\title{
Management Models in SMEs, Strategic Tool in Business Management
}

\section{Modelos Gerenciales en Las Pymes, Her- ramienta Estratégica en la Gestión Empresarial}

VII International Congress of

Science, Technology,

Entrepreneurship and

Innovation (SECTEI 2020)

Corresponding Author:

F. F. Esparza Paz

franqui.esparza@espoch.edu.ec

ffespaz@yahoo.es

Published: 26 August 2021

Production and Hosting by

Knowledge E

(c) L. R. Fiallos Ortega et al. This article is distributed under the terms of the Creative Commons Attribution License, which permits unrestricted use and redistribution provided that the original author and source are credited.
S OPEN ACCESS

\section{R. Fiallos Ortega ${ }^{1}$, F. F. Esparza Paz ${ }^{2}$, and A. G. Castelo Salazar ${ }^{3}$}

${ }^{1}$ Vicerrector de Investigación y Posgrado, Facultad de Ciencias Pecuarias, Escuela Superior Politécnica de Chimborazo, Riobamba, Ecuador

${ }^{2}$ Director Unidad de Educación a Distancia, Facultad de Administración de Empresas, Escuela Superior Politécnica de Chimborazo, Riobamba, Ecuador

${ }^{3}$ Docente Investigador, Facultad de Administración de Empresas, Escuela Superior Politécnica de Chimborazo, Riobamba, Ecuador

\section{Abstract}

The business sector constitutes an engine for the Ecuadorian economy through the generation of employment and redistribution of wealth. Therefore, given the current economic dynamics of high competition, globalization of markets, and use of information and communication technologies, business managers need to use different tools such as management models to achieve acceptable levels of utility, profitability, customer satisfaction and permanence in the consumer market. This research involved an analysis of the impact on corporate management of applying different management models of Small and Medium Enterprises (SMEs) in the city of Riobamba, province of Chimborazo (Ecuador). The study was descriptive, cross-sectional, exploratory and non-experimental and it was conducted in the first half of 2019. Data were collected through field investigation and the application of a structured survey with a sample composed of 136 managers, owners and administrators, from a population of 440 entrepreneurs. The results showed that $93 \%$ of entrepreneurs knew about the existence of management models, and $41 \%$ recorded that total quality is the most applied model followed by continuous improvement (18\%) and re-engineering (13\%). We conclude that entrepreneurs consider management models to be crucial in the management of their company.

Keywords: management model, business management, strategies, competitiveness, SMEs.

Resumen

El sector empresarial en todos sus niveles constituye un motor para la economía ecuatoriana, por la generación de empleo y redistribución de la riqueza; por ello los gerentes de las empresas dada la actual dinámica económica por la alta competencia, globalización de los mercados, uso de las tecnologías de información y comunicación, entre otras variables, necesita utilizar diferentes herramientas como son los modelos gerenciales para lograr niveles aceptables de utilidad, rentabilidad, satisfacción de los clientes y permanencia en el mercado consumidor. La investigación tiene como objetivo efectuar un análisis de la aplicación e incidencia en la gestión corporativa, de diferentes modelos gerenciales de las Pequeñas y Medianas empresas (Pymes) de la ciudad de Riobamba, provincia de Chimborazo (Ecuador). El estudio es descriptivo, transversal, exploratorio y no experimental efectuado en el primer semestre del año 2019, realizado mediante una investigación de campo, a través de la aplicación de una encuesta estructurada, seleccionando una muestra compuesta por 136 gerentes, propietarios y administradores, de una población de 440 empresarios. Los 
resultados obtenidos evidenciaron que el $93 \%$ de los empresarios conocen de la existencia de modelos gerenciales, el $41 \%$ registra que la calidad total es el modelo más aplicado seguido del mejoramiento continuo (18\%) y Reingeniería (13\%). Se concluye que los empresarios y emprendedores consideran a los modelos gerenciales de mucha importancia en la dirección de su empresa.

Palabras Clave: modelo gerencial, gestión empresarial, estrategias, competitividad, pymes.

\section{Introducción}

En un mundo de negocios globalizado y competitivo, por el uso de tecnologías de información y comunicación, diversidad de la producción, altos niveles de productividad, entre otros factores; los desafíos a los que se enfrentan en la actualidad los empresarios, administradores y gerentes de las pequeñas y medianas empresas (Pymes) hacen que sea indispensable la aplicación de modelos gerenciales, para poder vencer los retos y aprovechar las oportunidades de la industria. Las empresas en calidad de negocios privados se mantienen en los mercados competitivos si luego del proceso de gestión empresarial obtienen utilidades o ganancias y si estos beneficios son rentables en el largo plazo; las corporaciones pierden su oportunidad, capacidad de crecimiento y desarrollo sin la presencia de los beneficios de su labor productiva y comercial.

El grupo de empresas ubicadas en los estratos de pymes mantienen una gran importancia en la economía de las naciones y específicamente del Ecuador, pues permite generar una buena cantidad de plazas de trabajo tanto directas como indirectas, genera redistribución de la riqueza, sumado a que las mismas están relacionadas con todas las actividades económicas ya sea como productoras o proveedoras de artículos y servicios, lo que sustenta la producción nacional. Adicional a ello este conjunto de empresas han formalizado su gestión y su negocio cumpliendo con el pago de sus diferentes tributos (Impuesto a la Renta, Impuesto al Valor Agregado, etc.), y pudiendo acceder a contratos públicos con el estado en la construcción y/o provisión de servicios.

Teniendo en cuenta que el sector de las pymes se constituye en un motor de la economía del país, también adolece de ciertas dificultades, entre otras la falta de conocimientos y aplicación de modelos de gestión gerencial, que le permita emprender en el mejoramiento de procesos de desarrollo y crecimiento sostenibles, para su permanencia en el largo plazo en el mercado.

En este contexto la Facultad de Administración de Empresas, de la Escuela Superior Politécnica de Chimborazo, a través de su grupo de investigación y academia desarrolló con empresarios, gerentes y administradores de las pequeñas y medianas empresas 
(Pymes), de la ciudad de Riobamba, provincia de Chimborazo (Ecuador), un estudio con el fin de analizar los modelos de gestión empresarial que estos aplican en cada uno de sus sectores productivos comercial, de servicios o de manufactura y que posteriormente se puedan multiplicar en otro tipo de empresas.

La presente investigación tiene la siguiente estructura divididos en 4 apartados. En la sección de revisión de literatura se hace una exploración de los conceptos y marco teórico de la gestión empresarial, las habilidades y modelos gerenciales, como estos inciden e impactan en la dirección de las empresas pequeñas y medianas, así como en la competitividad empresarial. A continuación, se muestra la metodología de trabajo utilizada, en donde se indica que es un estudio descriptivo, no experimental y de campo, basado en el estudio de Monge y Zhunio efectuado en el 2013, mediante la aplicación de modelos gerenciales en establecimientos de alimentos y bebidas del centro histórico de Quito, método modificado para fines de esta investigación. En forma seguida se presentan los principales resultados y hallazgos encontrados en la exploración, así como las conclusiones, limitaciones y líneas de investigación futuras que podrían implementarse.

El objetivo de la presente investigación es efectuar un análisis de la aplicación y el impacto que tienen los modelos de gestión gerencial en las pequeñas y medianas empresas (Pymes) de la ciudad de Riobamba.

El objeto de estudio o problema u objeto de estudio tiene dos visiones en la investigación, por un lado, identificar los modelos gerenciales considerados por los empresarios, gerentes o administradores de las pymes, al momento de gerenciar una unidad productiva y por otra parte conocer si estos modelos considerados permitieron conseguir una dirección empresarial eficiente.

La revisión bibliográfica y documental permite reflexionar que los modelos gerenciales, se constituyen en una herramienta primordial para solucionar problemas en el espacio empresarial, pues mediante su aplicación se ha demostrado que los empresarios pueden obtener resultados positivos en la dirección de las empresas.

Bajo este ámbito, el problema de investigación puede formularse así: ¿Qué modelos gerenciales se utilizan y cómo el análisis de los modelos gerenciales incide en la gestión de las empresas pequeñas y medianas de la ciudad de Riobamba?

La hipótesis de la investigación planteada corresponde a: ¿Los modelos de gestión gerencial, permiten a los empresarios, gerentes o administradores lograr niveles de eficiencia en la dirección empresarial de sus unidades productivas, en la ciudad de Riobamba?

En calidad de estrategias de investigación el presente artículo proyecta el método estadístico, bajo la modalidad cuantitativa y el tipo de estudio descriptivo, ya que a través del uso de la estadística descriptiva se detalla el fenómeno estudiado, basados en una muestra aleatoria; sin llegar a una explicación o correlación del fenómeno u 
objeto de estudio. Siendo por tanto el alcance investigativo los modelos de gestión gerencial de empresarios.

\subsection{Revisión de literatura}

\subsubsection{Gestión gerencial y la competitividad empresarial}

En el ámbito empresarial una adecuada y eficiente gestión por parte de los empresarios, emprendedores, administradores o gerentes juega un rol importante en la consecución de los objetivos empresariales. En este contexto la gestión gerencial constituye el proceso aplicado por las empresas para orientar a sus diferentes unidades funcionales hacia la consecución de los objetivos y metas determinadas en cada una de ellas, a través de la aplicación de planes, programas, proyectos y actividades delimitados para certificar el correcto desarrollo de las operaciones y actividades, tanto desde el orden estratégico como táctico.

En este espacio según [1], 'La gerencia ha evolucionado como el hombre mismo, con cambios históricos de forma y de fondo, a partir de tres tipos y diferencias estructurales, dentro de las cuales están: según el enfoque, la orientación y, finalmente, según los mercados' (p. 61). Por ello [2], afirman que 'los entornos crecientemente dinámicos característicos de la sociedad del conocimiento demandan organizaciones cada vez más ágiles, flexibles y planas, con puestos de trabajo especializados y exigentes al tiempo que versátiles' (p. 26)

En este contexto, la competitividad es 'la capacidad de atraer, generar y/o desarrollas las capacidades y talentos necesarios en una empresa, para lograr altos niveles de productividad, que permitan a la empresa generar ventajas frente a la competencia' [3] (p. 138). Así mismo para [4], la competitividad empresarial, 'Es el proceso de obtención, análisis, interpretación y difusión de información de valor estratégico sobre la industria y los competidores, que se transmite a los responsables de la toma de decisiones en el momento oportuno' (p. 14).

De otra forma 'La competitividad está determinada por la productividad, definida como el valor del producto generado por una unidad de trabajo o de capital. La productividad es función de la calidad de los productos y de la eficiencia productiva' [5] (p. 4). Como lo confirman Kinra y Antai citado en [6], la competitividad es la habilidad de una organización para diseñar, producir y/o comercializar productos o servicios que tienen un nivel superior al de sus competidores, ya sean éstos tanto del mercado nacional como internacional, en términos de precio y cualidades del producto (p. 325).

En este contexto, para resolver los problemas de la competitividad empresarial debe contar con ciertos prerrequisitos para dar cumplimiento de la efectividad gerencial, algunos son: Acompañamiento total de la alta gerencia, distribución de poder, respaldo 
económico que sostenga lo que trace la gerencia, recursos técnicos, conciencia y disposición de todo el personal [7] (p. 4).

\subsubsection{Las habilidades gerenciales, mecanismos de cambio en la empresa}

Los gerentes de las empresas para el desempeño eficiente y eficaz de su gestión deben contar con ciertas habilidades o aptitudes. Las habilidades gerenciales constituyen el conjunto de competencias que las personas poseen para el cumplimiento de sus responsabilidades, a fin de realizar progresos en la unidad empresarial en procesos, productos y servicios, lo que permitirá a las empresas hacer frente a los variables cambios del mercado ayudando a mejorar la situación competitiva.

'Las empresas requieren de gerentes con múltiples conocimientos y una amplia gama de habilidades; pero, más que eso, exigen profesionales capaces de liderar grupos altamente capacitados' [8] (p. 116). Así y como lo describe [9], 'Las habilidades gerenciales son un conjunto de capacidades y conocimientos que el emprendedor debe poseer o desarrollar para realizar las actividades de administración y liderazgo en el rol de gerente o director de una organización' (p. 54). Es por ello que las habilidades que reclaman los gerentes tienen cambios constantes como consecuencia de las nuevas tendencias en el manejo de los negocios. Beltrán y Urrea [10] indica sobre las habilidades como: 'la capacidad para desempeñar una tarea física o mental; es la capacidad de una persona para hacer algo bien' (p. 21).

De otro modo y como lo explica en su investigación [11] 'las habilidades gerenciales son los medios con los cuales los gerentes traducen su propio estilo, herramientas, estrategias o técnicas favoritas a la práctica' (p. 123). Concluyendo se afirma que 'las Habilidades Gerenciales están interrelacionadas y sobrepuestas. Es difícil demostrar una sola habilidad aislada de las demás. Las habilidades no son conductas simples y repetitivas, son un conjunto integrado de respuestas complejas' [12] (p. 89). Así pues, 'Las características del entorno tanto mundial como nacional afectan directamente la actuación del gerente (...), ya que se les exige la formación de un individuo preparado para vivir en una nueva sociedad, caracterizada por incesantes e impredecibles transformaciones' [13] (p. 92).

En su investigación [14], manifiesta que: Un gerente eficaz está formado por tres componentes básicos; el saber hacer que se refiere a los conocimientos adquiridos a través de la formación; el querer hacer que involucra factores emocionales y motivacionales; y por último el poder hacer que hace referencia a la posición en la que se encuentra dentro de la estructura organizacional. Estos componentes conducen sin duda alguna a asegurar la mejor contribución de los directivos a los resultados esperados por la organización, es aquí donde los gerentes tienen que tener la capacidad de obtener lo mejor de los trabajadores, por lo que se hace cada día más imprescindible que todos 
los gerentes sean eficaces y estén dispuestos a asumir los desafíos del desarrollo organizacional (p. 15).

En definitiva, las empresas en la actualidad necesitan gerentes formados de manera profesional, con conocimientos, experiencia, habilidades, aptitudes y capacidades, que se constituyan agentes de cambio y transformación empresarial, en procesos, productos y servicios, con el objeto de que sean competitivos y se mantengan en el mercado.

\subsubsection{Los modelos gerenciales como instrumentos de gestión en las pymes}

La globalización económica y las rápidas transformaciones tecnológicas contribuyen a crear un ambiente ampliamente competitivo, en donde los principales beneficiados son los países desarrollados y las grandes empresas, mientras que los países en desarrollo y las PYMES enfrentan cada día mayores dificultades de crecimiento (...). [15] (p. 6)

'Las pequeñas y medianas empresas (PyME), son formas de organización empresarial que responden por la producción de una buena parte de los bienes y servicios en la sociedad contemporánea' [16] (p. 82).

El éxito de las empresas depende de diversos factores como capital, la posición en la etapa del ciclo de vida en la que se encuentre la empresa, el tiempo de recuperación del capital, pero también existen otros factores que influyen en su sostenibilidad de la empresa en el mercado competitivo [17] (p. 57). Por lo tanto, los gerentes de las pequeñas y medianas empresas necesitan de ciertos instrumentos de trabajo, para que su trabajo sea productivo, eficiente y efectivo, precisamente uno de ellos son los modelos de gestión gerencial. 'La necesidad de alcanzar un óptimo nivel de desarrollo empresarial, es posible a través del uso de determinados modelos gerenciales, los mismos que ayudan a entender y fortalecer cada uno de los diferentes procesos que se desarrollan en una empresa' [18] (p. 43).

Los modelos de gestión gerencial vienen adquiriendo cada vez mayor importancia en la dirección de las empresas sean pequeñas, medianas o grandes en las diversas actividades económicas en los cuales se desenvuelven, lo que anteriormente se aplicaban únicamente en grandes compañías. En este contexto los empresarios de las pymes deben aplicar mecanismos y herramientas de gestión que les permitan lograr sus objetivos empresariales: Utilidad, rentabilidad, crecimiento, diversificación y expansión de los mercados de consumo. La Tabla 1 muestra los diferentes modelos gerenciales que utilizan los empresarios de las pymes, y sus definiciones más importantes. 
Table 1

Definición de modelos gerenciales por varios autores.

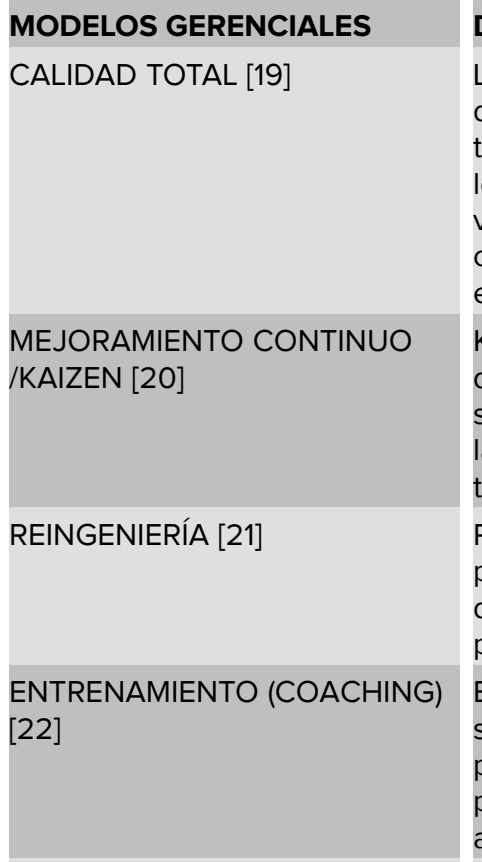

PLANIFICACIÓN ESTRATÉGICA [23]

COPIAR MEJORES PRÁCTICAS (BENCHMARKING) [24]

SUBCONTRATACIÓN

(OUTSOURCING) [24]

JUSTO A TIEMPO (JUST IN TIME) [25]

EMPODERAMIENTO (EMPOWERMENT) [26]

\section{DEFINICIÒN}

La calidad total abarca a toda la empresa y es un instrumento para su dirección. Pretende controlar, diseñar, medir y sugerir calidad en todas las funciones: Administrativas, comerciales, de producción, y en los aspectos externos e internos de la empresa, constituyéndose, a la vez, en una filosofía empresarial, permanente y de progreso constante, una renovación de la cultura de la empresa y un nuevo estilo de gestión.

Kaizen es un medio para mejorar continuamente en la vida personal, del hogar, la vida social y la vida laboral. En el área de trabajo, kaizen significa mejorar continuamente por cada uno de los empleados de las empresas (gerentes y trabajadores igual), en todo momento del trabajo.

Reingeniería es la revisión documental y el rediseño radical de los procesos para alcanzar mejoras espectaculares en medidas críticas del rendimiento, tales como costes, calidad, servicio y rapidez. El proceso se convierte en el proceso clave.

El coaching es la relación profesional y continua que ayuda a los sujetos a que obtengan grandes beneficios en su carrera, vida personal, organizaciones o negocios. Con la ayuda del coach, estas personas mejoran su calidad de vida, su desempeño y su aprendizaje.

La planificación estratégica es un proceso socio- político y científico orientado por objetivos de transformación cuanti - cualitativos de un sector particular de la realidad. Este proceso articula dos dimensiones de modo dialéctico: la reflexión y la acción, cuyo nexo lo constituye la evaluación, como proceso permanente de conocimiento, revisión, aprendizaje y retroalimentación. Entre la reflexión y la acción media la toma de decisiones, es decir definir sobre el qué y de qué manera se llevará algo a la práctica.

Permite diagnosticar, medir, comparar y evaluar entre otras cosas los servicios, procesos de trabajo, funciones, etc., facilitando el aprendizaje sobre uno mismo y los demás, enfocando el estudio de éstos últimos en cómo se prestan o realizan los servicios y no tanto en qué servicio se realiza o se presta.

Outsourcing es transferencia a terceros de actividades no medulares. En un contexto de globalización de mercados, las empresas deben dedicarse a innovar y a concentrar sus recursos en el negocio principal. Por ello la tercerización o Outsourcing ofrece una solución óptima y es motivo de permanentes alianzas estratégicas.

El justo a tiempo, es un conjunto integrado de actividades diseñadas para lograr un alto volumen de producción, utilizando inventarios mínimos de materia prima, trabajo en proceso y productos terminados. Las piezas llegan a la siguiente estación de trabajo justo a tiempo, se complementan y pasan por la operación rápidamente.

Empoderamiento se posibilita en tanto que la gente se problematiza, desarrolla una clara consciencia de las necesidades y de los recursos, de las posibilidades y de las limitaciones [...] pudiendo llegar a constituirse en una minoría disidente en la medida en que su labor autoafirmativa puede chocar con los intereses de instituciones, grupos o personas [...] empoderamiento es un proceso intencional, intersubjetivo y continuo de conversión de los individuos en sujetos conscientes de sí mismos, de las circunstancias y del entorno social, mediante la acción comprensiva, crítica y transformadora sobre sus propias interacciones sociales.

Fuente: Elaboración propia, a partir de varios autores, 2019. 


\subsubsection{Las estrategias empresariales en las pymes}

Las pequeñas y medianas empresas de cualquier actividad económica, ubicación geográfica, constitución legal, etc., en el desarrollo de sus actividades productivas tiene tres tipos de procesos, a saber: Estratégicos, operativos y de apoyo. Los tres se consideran importantes, sin embargo, los primeros (Estratégicos), son de mayor relevancia debido a que la alta dirección, administración, propietarios de las empresas son quienes generan las estrategias de corto, mediano y largo plazo para conseguir los objetivos empresariales: Utilidad y rentabilidad. Por ello [21], en relación con las estrategias afirman que 'El concepto de estrategia no tiene definición estándar y ha sido contemplado de maneras diferentes. Así, la estrategia se puede definir como un conjunto integrado de acciones destinadas a alcanzar una ventaja competitiva sostenible' (p. 383), entendido de esta forma la ventaja competitiva sostenible le permitirá mantenerse en un mercado tan competitivo y globalizado.

En este contexto, La estrategia se constituye en un aspecto muy importante en las decisiones que deben tomar las personas que tienen a cargo la gestión de una organización, en la que hay recursos de todo tipo que deben ser utilizados en forma óptima para cumplir con las políticas, objetivos y metas trazadas. Asimismo, la estrategia es una apuesta en un mundo globalizado en el que las empresas luchan por lograr mantenerse en el mercado utilizando todas las herramientas que poseen, estableciendo políticas flexibles y agresivas de gestión que les permitan posicionarse y tener continuidad en el futuro [27] (p. 153).

La estrategia corporativa abarca un enfoque planificado en el logro de los objetivos definidos: saber a dónde se quiere ir y como llegar allí. Los objetivos o metas significan 'el final del proceso' o 'el final del camino' y la estrategia significa 'los medios' o los ‘como' de una ‘implementación exitosa' [28] (p. 224).

Para que las estrategias de gestión que las empresas apliquen proporcionen los resultados deseados, es necesario que se cumplan entre otros los siguientes factores: Un enfoque sobre las metas estratégicas adecuadas, conducidas y defendidas por los directores, que nuclean a una organización detrás de la estrategia, determina las medidas e hitos del éxito y asegura que los recursos - financieros, tecnológicos y humanos, sean asignados eficazmente. La libertad concedida a todas las partes de la organización - individuos, equipos, distribuidores y socios estratégicos- para ser creativos y encontrar nuevos e innovadores modos de cumplir con estas metas [29] (p. 137).

De acuerdo con Pacheco [30], las estrategias que se utilizan con mayor intensidad por los empresarios de las pequeñas y medianas empresas, según su estudio se resumen en: 'Servicio al cliente, reducción de costos, mejoramiento continuo, innovación, diferenciación de productos, productividad y tecnología' (p. 196). 


\subsection{Materiales y Métodos}

\subsection{Tipo de estudio}

El estudio realizado en el primer semestre del año 2019 es carácter descriptivo, transversal, exploratorio y no experimental, a través de una exploración de campo mediante la aplicación de encuestas debidamente estructuradas a propietarios, gerentes y administradores de pequeñas y medianas empresas de la ciudad de Riobamba, provincia de Chimborazo (Ecuador).

\subsection{Población y muestra}

'Se habla de población o universo cuando se refiere a la totalidad, tanto de los sujetos seleccionados como del objeto de estudio' [31] (p. 88). La población está compuesta por 440 empresarios de las pymes de Riobamba- Chimborazo, en las diferentes actividades económicas (Clasificación Internacional Industrial Uniforme CIIU: Confecciones, textiles, metalmecánicas, automotrices, madereras, gráficas, alimenticias, químicas, entre otras), registradas al año 2018 en el catastro de la Unidad de Rentas del Gobierno Autónomo Descentralizado del cantón Riobamba (GADMCR).

La muestra según [32], 'Es la parte de la población que se selecciona, de la cual realmente se obtiene la información para el desarrollo del estudio y sobre la cual se efectuarán la medición y la observación de las variables objeto de estudio' ( $p$. 165). Para calcular el tamaño de la muestra se utilizó la fórmula de [33] (p. 227), bajo un error muestral del 7\%, un nivel de confianza del $95 \%, Z=1,96$ y $P=Q=0,50$; consecuentemente se efectuaron 136 encuestas a empresarios.

\subsection{Proceso de la investigación}

Como principal instrumento de recolección de información se utilizó una encuesta con dos apartados (Datos generales e información del uso de modelos gerenciales), a través de preguntas cerradas, mediante el uso de la escala de Likert (1932). Adicional se aplicó el método de muestreo probabilístico, que de acuerdo con [33], 'Este tipo de muestreo alcanza mayor rigor científico, pues cumple con el principio de equi-probabilidad; según el cual todos los elementos de la población tienen la misma probabilidad de ser elegidos de una muestra' (p. 223). La tabulación y procesamiento de datos se efectuó con el uso de la herramienta informática estadística SPSS (Statistical Packge for Social Sciences), lo que permitió sistematizar la información en tablas y figuras. 


\section{Resultados y Discusión}

\subsection{Características generales de empresarios de las Pequeñas y medianas empresas}

La Tabla 2 muestra los principales hallazgos relacionados a las características o rasgos generales de los empresarios, gerentes y administradores de las pequeñas y medianas empresas de la ciudad e Riobamba.

\section{Table 2}

Características de los empresarios de las Pymes.

\begin{tabular}{|c|c|}
\hline CARACTERÍSTICAS & PRINCIPALES HALLAZGOS \\
\hline Género & $\begin{array}{l}\text { El } 71 \% \text { de los empresarios, pertenecen al género masculino, al género } \\
\text { femenino el } 29 \% \text {. Existe importante participación del género } \\
\text { femenino en actividades empresariales. }\end{array}$ \\
\hline Edad & $\begin{array}{l}\text { En las pymes el } 39 \% \text { de los empresarios se encuentran entre los } 36 \text { y } \\
45 \text { años, el } 22 \% \text { se sitúa entre los } 26 \text { y } 35 \text { años, así como entre } 46 \text { y } \\
55 \text { años, respectivamente. El promedio de edad para administrar las } \\
\text { pymes es } 40 \text { años. }\end{array}$ \\
\hline Instrucción Académica & $\begin{array}{l}\text { Los empresarios de las pymes han cursado estudios de bachillerato } \\
(69 \%) \text {, las personas que poseen título de tercer nivel representan el } \\
21 \% \text {. En los empresarios se puede evidenciar un bajo nivel de } \\
\text { preparación académica, apenas han estudiado el nivel medio, lo que } \\
\text { afecta en el desarrollo de sus actividades empresariales. }\end{array}$ \\
\hline $\begin{array}{l}\text { Tiempo en la actividad } \\
\text { económica }\end{array}$ & $\begin{array}{l}\text { Los empresarios que gerencian las pymes hasta } 3 \text { años representan } \\
\text { el } 40 \% \text {, un } 29 \% \text { entre } 4 \text { y } 7 \text { años, y el } 25 \% \text { entre } 11 \text { y } 15 \text { años. Se nota } \\
\text { claramente que existe muy poco tiempo en la administración } \\
\text { empresarial. }\end{array}$ \\
\hline $\begin{array}{l}\text { Número de empleados por } \\
\text { empresa }\end{array}$ & $\begin{array}{l}\text { El } 73 \% \text { de las pymes registran hasta } 10 \text { empleados en sus nóminas, } \\
\text { entre } 11 \text { y } 20 \text { empleados el } 20 \% \text {. Se puede observar que las pymes } \\
\text { no ocupan mayor cantidad de mano de obra. }\end{array}$ \\
\hline
\end{tabular}

Fuente: Propia del autor a partir de encuestas aplicadas a empresarios de las Pymes, 2019.

Los hallazgos del estudio indican que las pymes se desarrollan en varias actividades productivas, como alimentos, confecciones, cuero, calzado, maderas, metalmecánicas, gráficas, entre otras; existiendo mayor participación del género masculino en la gestión y administración de las empresas, constituyéndose en tendencia en el sector empresarial del Ecuador. En calidad de unidad productiva compite con otras corporaciones que procesan y comercializan productos y/o servicios similares o sustitutos, actúan en un entorno donde los recursos materiales, económicos, tecnológicos y el talento humano de calidad escasean.

La edad de los empresarios se considera en condición adecuada debido que existe la experiencia apropiada para una correcta dirección y toma de decisiones. Los empresarios de las pymes demuestran una baja preparación académica por la falta de instrucción formal, la mayor parte de propietarios y gerentes han cursado el nivel secundario de educación. El tiempo que llevan los empresarios de las pymes en la administración de 
su empresa permite a las empresas mantener una estabilidad en el mercado, en su gestión productiva y comercial.

Se evidencia por otro lado la existencia de una baja nómina de empleados y trabajadores en cada una de las pymes, notándose la falta de contratación permanente de personal para sus diferentes actividades. La responsabilidad de la dirección y gestión empresarial de cada una de las pymes está bajo la conducción del propietario, su esposa o viceversa, lo que en cierto modo garantiza la aplicación de los diversos modelos, así como la consecución de los resultados corporativos.

\subsection{Los modelos gerenciales en la gestión de las pymes}

Table 3

Administración de las pequeñas y medianas empresas.

\begin{tabular}{l|l|l|l}
\hline TIPO DE ADMINISTRADOR & RESPUESTAS & $\%$ & \% ACUMULADO \\
\hline Propietario (a) - esposa (o) & 116 & 85,29 & 85,29 \\
\hline Gerente & 8 & 5,88 & 91,17 \\
\hline Administrador & 12 & 8,83 & 100,00 \\
\hline TOTAL & 136 & 100,00 &
\end{tabular}

Fuente: Propia del autor a partir de encuestas aplicadas a empresarios de las Pymes, 2019.

La Tabla 2 permite observar que él o la propietario (a) y su esposa (o), son responsables por la gestión y administración de la pequeña o mediana empresa, pues alrededor del $85 \%$ lo ratifican, por el contrario apenas un 15\% de las pymes están bajo la dirección tanto de un gerente como de un administrador, lo que corrobora que las pequeñas y medianas empresas (Pymes), tienen la responsabilidad en sus propietarios, pues cuidan los diferentes recursos que ponen en riesgo en su actividad económica. Por ello se puede manifestar que, 'El sector de las pymes en el Ecuador en la mayoría de los casos constituye en negocios familiares cuyo recurso financiero procede del mismo núcleo familiar, siendo administradas por sus propios dueños' [34] (p. 11).

\section{Table 4}

Conocimiento de los modelos de gestión empresarial.

\begin{tabular}{l|l|l|l}
\hline OPCIÓN & RESPUESTAS & $\%$ & \% ACUMULADO \\
\hline $\mathrm{Si}$ & 126 & 92,65 & 92,65 \\
No & 10 & 7,35 & 100,00 \\
\hline TOTAL & 136 & 100,00 &
\end{tabular}

Fuente: Propia del autor a partir de encuestas aplicadas a empresarios de las Pymes, 2018.

Los modelos de gestión gerencial son importantes en el desarrollo de las actividades económicas y en cumplimiento de objetivos corporativos, por ello y en relación con el 


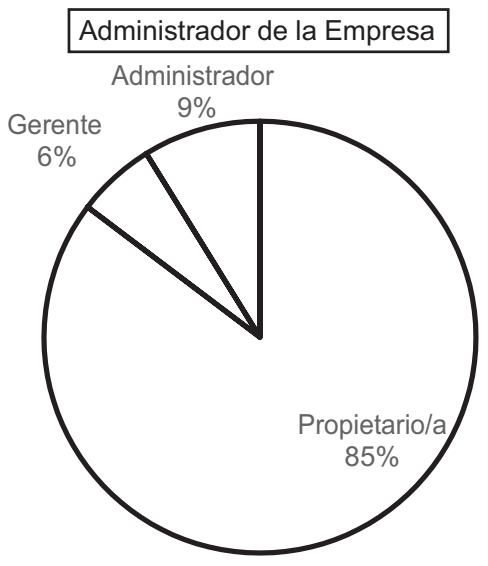

Figure 1

Resultados de la Tabla 2.

conocimiento de los modelos de gestión gerencial que puede utilizar los gerentes de las pymes para mejorar la gestión corporativa, la Tabla 3 muestra que los empresarios conocen de la existencia de los diversos modelos (93\%), el 7\% restante lo desconocen.

'Los modelos gerenciales son estrategias de gestión organizacionales que se utilizan en la dirección y el desarrollo del sistema, así como en todos sus procesos' [35] (31). Por ello, de acuerdo con Pérez y Nieto, citado por [36], los modelos de gestión gerencial fueron creados 'con el propósito de facilitar la modernización gerencial de las empresas hacia organizaciones modernas, competitivas, centradas en la innovación y capaces de competir en un mundo global' (p. 24).

\section{Table 5}

Aplicación de modelos gerenciales en las Pymes.

\begin{tabular}{llll}
\hline TIPO DE MODELO GERENCIAL & RESPUESTAS & $\%$ & \% ACUMULADO \\
\hline Calidad Total & 56 & 41,18 & 41,18 \\
\hline Mejoramiento continuo & 25 & 18,38 & 59,56 \\
\hline Reingeniería & 18 & 13,24 & 72,80 \\
\hline Entrenamiento (Coaching) & 14 & 10,29 & 83,09 \\
\hline Planificación Estratégica & 10 & 7,35 & 90,44 \\
\hline $\begin{array}{l}\text { Copiar mejoras prácticas } \\
\text { (Benchmarking) }\end{array}$ & 6 & 4,41 & 94,85 \\
\hline Subcontratación (Outsourcing) & 4 & 2,94 & 97,79 \\
\hline Justo a Tiempo (Just Time) & 2 & 1,47 & 99,26 \\
\hline Empoderamiento (Empowerment) & 1 & 0,74 & 100,00 \\
TOTAL & 136 & 100,00 &
\end{tabular}

Fuente: Propia del autor a partir de encuestas aplicadas a empresarios de las Pymes, 2019.

Las empresas pequeñas y medianas buscan a través de la aplicación de modelos gerenciales obtener sus objetivos empresariales, es por ello que la calidad total con 
el $41 \%$ de participación es el modelo gerencial más utilizado, que obedece a que las empresas procuran generar productos y servicios de calidad; concomitante el mejoramiento continuo (18\%) es el segundo modelo más utilizado, seguido de la Reingeniería (13\%) y del entrenamiento (10\%); que en conjunto suman el $83 \%$. El empoderamiento $(0,74 \%)$ y Justo a Tiempo $(1,47 \%)$, son los modelos gerenciales menos utilizados, por cuanto debido a que las empresas ven complicado la aplicación y uso de esos modelos.

\section{Table 6}

Importancia de los Modelos Gerenciales en las pequeñas y medianas empresas.

\begin{tabular}{l|l|l|l}
\hline CONSIDERACIÓN & RESPUESTAS & $\%$ & \% ACUMULADO \\
\hline Muy importante & 108 & 79,41 & 79,41 \\
\hline Importante & 14 & 10,29 & 89,70 \\
\hline Neutral & 7 & 5,15 & 94,85 \\
\hline Poco importante & 2 & 1,48 & 96,33 \\
No es importante & 5 & 3,67 & 100,00 \\
\hline TOTAL & 136 & 100,00 &
\end{tabular}

Fuente: Propia del autor a partir de encuestas aplicadas a empresarios de las Pymes, 2019.

Los empresarios de las pymes aplican modelos de gestión gerencial, eso significa la importancia que se le da a estas herramientas de dirección, en la Tabla 5 se puede evidenciar que el $79 \%$ y el $10 \%$ de los empresarios consideran como muy importantes e importantes respectivamente el uso de los diversos modelos, que en acumulado generan aproximadamente el $90 \%$. Apenas un $5 \%$ considera como poco y no importante la utilización de modelos gerenciales en el desempeño de las empresas.

Table 7

Calificación de uso de los Modelos Gerenciales en las Pymes.

\begin{tabular}{l|lll}
\hline OPCIÓN & RESPUESTAS & $\%$ & \% ACUMULADO \\
\hline Excelente & 96 & 70,59 & 70,59 \\
\hline Muy bueno & 14 & 10,29 & 80,85 \\
Bueno & 19 & 13,97 & 94,85 \\
Malo & 7 & 5,15 & 100,00 \\
\hline TOTAL & 136 & 100,00 &
\end{tabular}

Fuente: Propia del autor a partir de encuestas aplicadas a empresarios de las Pymes, 2019.

En párrafos anteriores se describió que la aplicación de los modelos de gestión gerencial gira alrededor del $90 \%$ de importantes, por tanto, su aplicabilidad genera resultados favorables para las pequeñas y medianas empresas, el 71\% lo considera de excelente seguido del 10\% como muy buena, esto demuestra la relevancia de su aplicación en las pymes. 


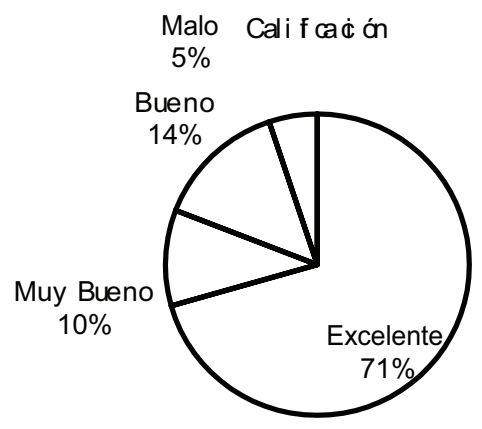

Figure 2

Resultados obtenidos en la Tabla 6.

En definitiva, y como lo manifiestan [37], la complejidad del fenómeno de la globalización demanda de las organizaciones la adopción de modelos gerenciales que permitan competir en los mercados internacionales, con su consecuente adaptación al comportamiento de nuevos consumidores, así como al manejo de otras formas en los procesos de comercialización y distribución (p. 28).

\section{Conclusiones}

La Escuela Superior Politécnica de Chimborazo, a través de la Facultad de Administración de Empresas, como institución de educación superior, entre uno de sus cuatro ámbitos de acción promueve la investigación y la vinculación con la sociedad, mediante el desarrollo de estudios en beneficio de los diferentes sectores económicos y productivos; es por ello por lo que se da cumplimiento a estos aspectos de la institucionalidad universitaria.

A través del estudio y a la luz de los hallazgos ubicados se puede colegir que el objetivo y la hipótesis planteada fueron comprobadas a través del estudio investigativo, los empresarios han confirmado que los modelos de gestión gerencial son muy importantes, que ayudan en la dirección de sus empresas, mejorando los indicadores de crecimiento y desarrollo de sus pymes.

Con el estudio se demuestra que los empresarios y emprendedores de las pymes conocen de la existencia de los modelos gerenciales, así como definen cuales son los más utilizados y que demuestran una importancia absoluta en la gestión corporativa, adicional que los califican como muy buenos en su aplicabilidad, que les ayudan a conseguir los objetivos empresariales, siendo los más importantes la calidad, el mejoramiento continuo y la planificación estratégica. Los hallazgos definidos que se alcanzaron han sido presentados de forma sencilla y resumida en tablas y figuras, siendo de fácil comprensión y lectura. 
Los modelos de gestión gerencial representan por tanto un eje fundamental para la gerencia empresarial moderna, los sistemas industriales, la mecanización y estandarización de los procesos productivos y la eficiencia administrativa y operativa en el uso de los diversos recursos corporativos, si bien en su momento representaron estrategias organizacionales que permitieron la optimización empresarial, hoy han quedado rezagados con la aplicación de los modelos gerenciales.

El estudio plantea como limitaciones trascendentes que fue realizado en un momento único de tiempo, lo cual solo permite tener datos únicamente como diagnóstico momentáneo, sería importante que se hagan los estudios en diferentes momentos, con el fin de medir los cambios que existe en el comportamiento de los emprendedores de las pymes al tratar el tema de la aplicación de modelos de gestión gerencial.

Se podrían realizar otros estudios en otros segmentos productivos, así como en otras actividades económicas, sean estas de comercio o servicios, agropecuarias, entre otras. Un buen espacio de investigación que se podría aplicar en épocas de crisis, como las que el sector empresarial del Ecuador está viviendo, como es el caso de la pandemia por el aparecimiento del Covid-19, un virus que afecta de manera ostensible y global a nivel no solo de nuestro país sino del mundo, a nivel social, económico, de salud, y principalmente a los diferentes sectores productivos.

El tema de investigación, debido a su relevancia permite considerar una base científica que puede servir para fututos estudios, que pueden desarrollar otros investigadores del tema de la aplicación y uso de modelos gerenciales, permitiendo efectuar una comparación por diferentes sectores económicos, por clase de empresa, entre otras variables consideradas.

\section{Agradecimiento}

Los autores de la presente investigación dejamos constancia de agradecimiento a los estudiantes del primer semestre de la Escuela de Administración de Empresas, de la Facultad de Administración de Empresas, Escuela Superior Politécnica de Chimborazo, por su excelente trabajo en la recopilación de datos mediante la aplicación de encuestas. Esperamos que sea de mucha utilidad en la formación estudiantil y académica.

Además, a los empresarios, gerentes o administradores de las pequeñas y medianas empresas de la ciudad de Riobamba, por la colaboración demostrada durante el proceso investigativo. 


\section{Conflicto de Intereses}

No existe conflicto de intereses entre los autores, de acuerdo con la responsabilidad y distribución del trabajo realizado. Adicional con los empresarios propietarios de las pequeñas y medianas empresas.

\section{References}

[1] Castillo J. Gerencia del Siglo XXI. Revista Escuela de Administración de Negocios. 2005;54:59-83.

[2] Zarmeño L, Armenteros M, Sologaistoa A, Villanueva Y. Competencias directivas: Su identificación para instituciones de educación superior. Revista Global de Negocios. 2014;2:25-42.

[3] Villarán K. Plan de Negocios. Herramienta para evaluar la viabilidad de un negocio. Lima: USAID, MYPE COMPETITIVA; 2009.

[4] Escorsa P. Vigilancia tecnológica e Inteligencia Competitiva. Asociación Multisectorial de Empresas; 2008.

[5] Porter M. La competitividad en el desarrollo empresarial. España: Difunda ediciones; 2017.

[6] Pesantez R, Orellana D, Toral A. El capital humano como fuente de innovación y su impacto en la competitividad empresarial. Medellín: Editorial Corporación CIMTED; 2018.

[7] Leyva A, López J, Nuño J, Cavazos J. Competencias gerenciales en la competitividad empresarial de las PyME's. Revista INVURNUS. 2014;9:3-11.

[8] Naranjo R. Habilidades gerenciales en los líderes de las medianas empresas de Colombia. Revista Pensamiento \& Gestión. 2015;38:119-146.

[9] Griffin R. Economía de la Defensa. Escuela naval militar, Publicación 547. Plan General de Contabilidad Pública; 2017.

[10] Beltrán N, Urrea D. Diseño e implementación del modelo de gestión por competencias y evaluación del personal según el modelo, para la empresa aportes en línea [Tesis de Especialización en Gestión Humana]. Universidad Escuela de Negocios EAN, Bogotá DC.; 2013.

[11] Hernández A, Alemán A, Soto J, Aispuru J. Gestión y Administración Militar, Escuela Naval Militar; 2016.

[12] Illera R. Administración y funciones de empresa. Madrid: Editorial Sanz y Torres, Madrid; 2014.

[13] Castro L, Fossi L, Guerrero W, Vera L. Competencias gerenciales y gestión de los proyectos educativos. Orbis. Revista Científica Ciencias Humanas. 2013;9: 91-108. 
[14] Reyes K. Habilidades gerenciales y desarrollo organizacional. Estudio realizado con gerentes de los hoteles inscritos en la Asociación de Hoteles de Quetzaltenango. [Tesis de Pregrado para obtener el título de Licenciada en Psicología Industrial]. Guatemala: Universidad Rafael Landívar; 2016. P. 1-90.

[15] Vargas J, Soto J, Rosas K. La relación del compromiso organizacional, con el nivel de estudios, la edad y el género en trabajadores de la industria de calzado en el León, Guanajuato. Observatorio Iberoamericano del Desarrollo Local y la Economía Social. Revista Grupo EUMED.NET. 2014;8.

[16] Carrión L, Zula J, Castillo L. Análisis del modelo de gestión en pequeñas y medianas empresas y su aplicación en la industria del catering en Ecuador. 2016;82-102.

[17] Vallejo M, Altamirano F, Arias J. Valores del emprendedor y su efecto en la sostenibilidad en la pymes. Revista Mktdescubre. 2016;7(6):56-68.

[18] Monge E, Zhunio B. Aplicación de modelos gerenciales en los establecimientos de alimentos y bebidas del centro histórico de Quito. Revista RICIT. 2013;5:40-68.

[19] Pérez M. Calidad total en las administraciones públicas. Málaga: ICB; 2011.

[20] Carreras M. Creatividad emprendimiento y mejora conitua. Barcelona: Reverté; 2019.

[21] Mallo C, Merlo J. Control de gestiòn y control presupuestario. Madrid: McGrawhill; 1995.

[22] Pérez M. Coaching. Málaga: ICB’ 2017.

[23] Rodríguez M. Planificación estratégica: Fundamentos y herramientas de actuación. Córdova: Brujas; 2016.

[24] Mora F. Outsourcing \& Benchmarking. Santa Fe, Argentina: El Cid; 2009.

[25] Chase R. Administración de producción y operaciones: Manufactura y Servicios. Santa fe de Bogotá: Mc Graw Hill; 2000.

[26] Pérez G. Conflictividad y empoderamiento: En agrupaciones sociales y contemporáneas. Guadalajara: Instituto Tecnológico y de Estudios Superiores de Occidente; 2015.

[27] Contreras E. El concepto de estrategia como fundamento de la Planeación Estratégica. Revista Pensamiento \& Gestión. 2013;35:152-181.

[28] Vause B. Análisis estratégico de compañías. The Economist. 2008.

[29] Syrett M. Estrategia de negocio, como dar en el blanco. The Economist. 2010.

[30] Pacheco C. Estrategias empresariales más utilizadas por las pymes en Sincelejo. Revista Económicas CUC. 2013;34(1):183-202.

[31] Del Cid A, Méndez R, Sandoval F. Investigación: Fundamentos y Metodología. 2nd edition. México: Editorial Pearson Educación; 2011.

[32] Bernal C. Metodología de la Investigación. Para administración, economía, humanidades y ciencias sociales. 3th edition. México: Pearson Educación; 2016. 
[33] Vara-Horna A. Desde la idea hasta la sustentación: Siete pasos para una tesis exitosa. Un método efectivo para las ciencias empresariales. Instituto de Investigación de la Facultad de Ciencias Administrativas y Recursos Humanos. 3rd edition. Lima-Perú: Universidad de San Martín de Porres; 2012.

[34] Santo D. (2019). Factores determinantes en la toma de decisiones de financiamiento a largo plazo en el sector de la construcción en la provincia de Cotopaxi [Tesis de posgrado]. Guayaquil: Universidad Técnica de Ambato; 2019.

[35] Montealegre J, Delgado A, Cubillos C. Relaciones entre modelos gerenciales y pensamiento

estratégico empresarial en las empresas agroindustriales del departamento del Tolima. Pensamiento y gestión. 2017;42:26-47.

[36] Tobar C. La aplicación de la teoría de las restricciones, como modelo de gestión en pequeñas y medianas empresas. Ingfam. 2014;1: 23-34.

[37] Gutiérrez J, Concha J. Modelo gerencial de la empresa exportadora colombiana: análisis de decisiones, estrategia y geoconocimiento. Revista Lebret. 2014;6:323347. 